\title{
Towards Provenance Aware Comment Tracking for Web Applications
}

\author{
James R. Michaelis and Deborah L. McGuinness \\ Tetherless World Constellation \\ Rensselaer Polytechnic Institute \\ Troy, NY 12180 \\ \{michaj6,dlm\}@cs.rpi.edu
}

\begin{abstract}
Provenance has been demonstrated as an important component in web applications such as mashups, as a means of resolving user questions. However, such provenance may not be usable by all members of a given applications user base. In this paper, we discuss how crowdsourcing could be employed to allow individual users to get questions answered by the greater user base. We begin by discussing a technologyagnostic model for incorporating Provenance Aware Comment Trackers (PACTs) into web applications. Following this, we present an example of a PACT-extended application with accompanying two accompanying use cases.
\end{abstract}

\section{Introduction}

On the Web, applications are continuously being created to serve communities of users. These applications are capable of generating browsable content via an interface for users to review and browse. In reviewing this content, users may have questions about the content they see, which could impact their trust in the application [1.

Provenance can provide information on the creation and history of artifacts, such as web-based resources [8] and be used as an aid in question answering. However, for someone to work effectively work with the provenance of web content, certain kinds of background are necessary. Examples of such background include understanding how the individual components of an application's workflow (e.g., code, supporting technologies) are supposed to function [7. Personalized views of provenance can help with this 2,12 . Yet these approaches are not guaranteed to produce understandable provenance for a given user in all cases. While such a user may be unable (or unwilling) to review this provenance, other members of a web community may be able to it for them. The use of collective intelligence, or crowdsourcing, has been applied previously in web applications [3]. Due to differences in the backgrounds of individuals (i.e., different knowledge and experiences), certain users may understand application output that a particular user doesnt. As such, access to the insights of a greater community of users for a web application could be a valuable aid to any single community member. Here, it 
is important to emphasize the role of provenance in crowdsourcing driven question answering. For web applications based on data and services that continually change (e.g., an application that pulls in content from Wikipedia), what one user sees in the interface may be quite different from what other users see. As such, provenance can provide context to user questions on web applications, such that other users can make sense of them.

\subsection{Deliverables}

This work discusses the idea of crowdsourcing provenance evaluation as a means of addressing user questions on web application outputs, and is intended as a starting point upon which more applied work can later follow. To help convey our current views, three deliverables are provided in this paper:

- A model for extending web applications with provenance-based comment tracking, known as the Provenance Aware Comment Tracker (PACT) model. In the PACT model, users may comment on sections of a web application interface, known as outputs. In turn, other web community members may reply to this user, forming discussion threads in the process.

- An example of a PACT-extended web application, titled US Global Aid. This application presents a mashup of user foreign aid contributions made to individual countries by the US Agency for International Development (USAID) with supplementary country facts provided by DBPedia.

- Based on this example application, two use cases are presented to illustrate PACT usage.

Additionally, it is important to mention what this work doesn't attempt to cover:

- User interface or usability issues for accessing a PACT from a web application, or presenting provenance and presenting previously made comments to end users.

- The usage of specific technologies for implementing PACTs in web applications. However, some recommendations on promising technologies are provided for this purpose at the end of the paper.

The outline of the remainder of the paper is as follows: Section 2 discusses related work; Section 3 introduces the PACT model; Section 4 introduces the US Global Aid mashup, and discusses corresponding use cases; finally, Section 5 provides our discussion and concluding remarks.

\section{Related Work}

The use of provenance in mashups has received significant recent attention. For instance, the documenting of application activities, based on models such as the p-structure [5], has been explored. Additionally, frameworks for tracking the provenance of mashup evolution, such as VISMASHUP [10, have been developed. Here, users can view multiple instances of a mashup, distinguished by 
the provenance of their development by an end user. This work builds on the VisTrails [4] approach of tracking the provenance of workflow evolution.

Likewise, the application of crowdsourcing toward problem solving has received significant attention [3. Examples of the kinds of problem solving explored include reviewing the quality of Wikipedia articles [6] and linguistic annotation [13. In both of these particular cases, communities of users are assigned tasks, and complete them through the Amazon Mechanical Turk 1 service. To our knowledge, little work has been explored on applying crowdsourcing of any kind toward provenance evaluation.

\section{The Provenance Aware Comment Tracker (PACT) Model}

In this section, we discuss the PACT model model and its relationship to web applications. Here, we assume the existence of:

- An application A consisting of a workflow $W=\{N, C, I\}$ with:

- $\mathbf{N}$ : A set of all input parameters to the application, where $|\mathrm{N}| \geq 0$.

- P: A set of all processing stages carried out by the application, where $|\mathrm{P}| \geq 0$.

- I: The interface of the application, consisting of a set of outputs viewable by a user 2 , where $|\mathrm{I}|>0$.

- A user community $\mathrm{U}$, where $|\mathrm{U}| \geq 0$.

Additionally, we assume that each user $\mathrm{u} \in \mathrm{U}$ is capable of running $\mathrm{A}$, resulting in an execution of W. For each execution of $\mathrm{W}$ :

- The same set of input parameters is used, with possibly changing values (e.g. if one of the parameters is an RSS feed).

- The same set of processing stages $\mathrm{P}$ are used, with possible variability in execution sequence (based on factors such as variability in the input parameters).

- The same set of interface outputs are displayed, with possible variability based on the execution sequence.

What this means is that while each execution of the application will follow a common plan, different users may end up seeing different information in the application outputs. With these assumptions in mind, we define the components of a PACT based on the activities an end user can do for each application output:

- Inspect Provenance: A provenance trace detailing all activities managed by the application to generate the output should be accessible (i.e. the trace goes from the output to input parameters), should a user want to inspect provenance themselves.

\footnotetext{
${ }^{1}$ https://www.mturk.com/

${ }^{2}$ Here, we are not attempting to define what constitutes an application output. For the purpose of this paper, it is some discrete entity whose provenance is logged.
} 
- Make a comment: Additionally, for each application output, a user should be able to make a comment based on what they see. For each comment made, the PACT should register the following things, in addition to the comment itself: (i) information on the commenting user (e.g., name and email address), (ii) a provenance trace of the output they saw, going from the output to inputs, and (iii) an glimpse of the output as the user saw it, which could either be an image or a fully functional output reproduced for others to look at. These materials can help establish the context of a given user's comments for the greater web community.

- Review comments: Here, members of the web community can review comments made by given users. For each comment, the users will be able to see the comments, as well as three types of information described above.

- Reply to a comment: Upon reviewing a comment, users can reply to comments - yielding child comments or to the children of comments yielding discussion threads based on an original comment.

\section{Exemplar Application: US Global Aid}

Mashups are a kind of web-based application designed to integrate data and web services from different sources, yielding new functionality in the process. These kinds of web applications are particularly good candidates for provenance usage as it can often be unclear what data sources used to create the information presented in the interface [8]. To illustrate the use of PACTs in web applications, we present a basic mashup called US Global Aid3. This mashup, shown in Fig. 1. is designed leverage Semantic Web based resources to display two kinds of information:

- Foreign aid contributions made to individual countries in 2008 by the US Agency for International Development (USAID), derived from data hosted by the US Government website Data.gov 4

- Facts for individual countries obtained from DBPedia.org. Here, population, Human Development Index (HDI) and Failed State Index (FSI) are presented5.

To ensure the user community views this mashup as reliable, it needs to have mechanisms to preserve transparency, as well as the accountability of data it uses. This helps address two general issues: first, flaws may be present in mashup workflow itself that misrepresent content; second, the mashup output may change regularly due to changes in data sources.

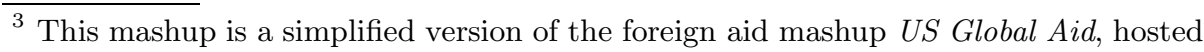
at http://www.data-gov.tw.rpi.edu/

${ }^{4}$ Specifically, Dataset 1554 is referenced: http://www.data.gov/raw/1554

${ }^{5}$ On DBPedia, these may be referenced for individual countries through the RDF properties dbpprop:populationEstimate, dbpprop:hdi and dbpprop:fsi respectively, where the namespace dbpprop is defined as http://dbpedia.org/property/ 


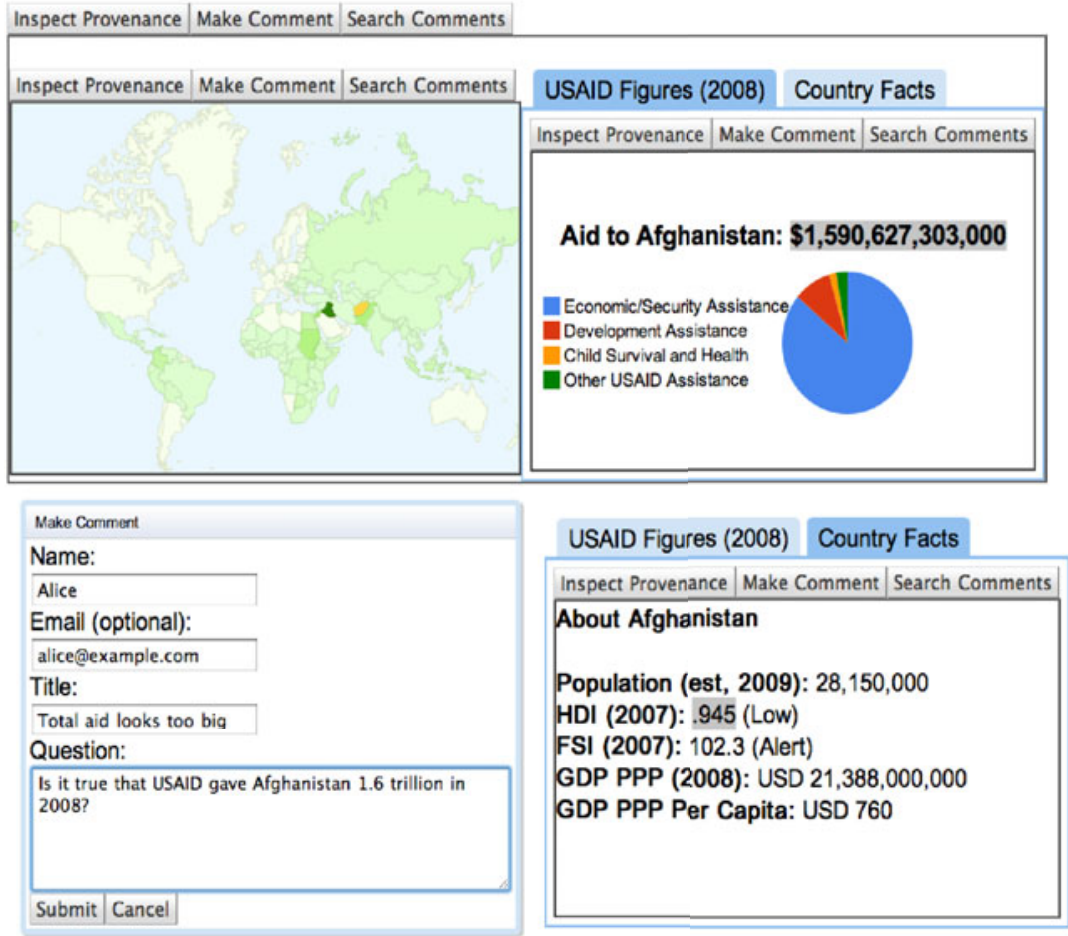

\begin{tabular}{|c|c|c|c|c|c|}
\hline \multicolumn{6}{|c|}{ Search Comments } \\
\hline Title & User & Comment & Replies & Provenance & Output \\
\hline $\begin{array}{l}\text { Total aid } \\
\text { looks } \\
\text { too big }\end{array}$ & Alice & $\begin{array}{l}\text { Is it true that USAID gave } \\
\text { Afghanistan } 1.6 \text { trillion in } \\
2008 ?\end{array}$ & View & Inspect & 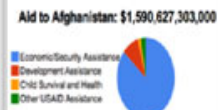 \\
\hline $\begin{array}{l}\text { HDI } \\
\text { looks } \\
\text { too high }\end{array}$ & Carol & $\begin{array}{l}\text { How could Afghanistan } \\
\text { have an HDI of .945 in } \\
2007 \text { (when the US was } \\
.956) ?\end{array}$ & View & Inspect & 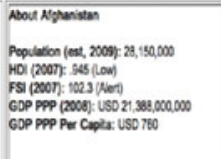 \\
\hline
\end{tabular}

Fig. 1. Screenshots from the mashup US Global Aid. Top: A world map, shaded according to the amount of aid received by individual countries, along with aid figures for Afghanistan. This are the outputs seen by Alice in Use Case 1, in which Afghanistan is shown as receiving about 1.6 trillion from USAID. Middle Left: The "Make Comment" form that Alice uses to make her comment in Use Case 1 Middle Right: Country facts for Afghanistan that Carol Sees in Use Case 2, in which an HDI value of .945 is given. Bottom: The "Search Comments" interface used by Bob to find, and reply to, Alice and Carol's comments in Use Cases 1 and 2. Here, the search interface is displaying comments for the two different outputs that were commented on. 


\subsection{Use Case 1: Problem in Mashup}

Here, a user Alice runs the mashup and sees that Afghanistan received about 1.6 trillion in 2008 - an amount which would constitute a significant portion of the US national debt 6 . Alice has little background with what provenance is, so she decides to simply make the comment: "Is it true USAID gave Afghanistan 1.6 trillion in 2008?". Following this, another user Bob decides to search for comments made on the Afghanistan figures and finds Alice's comment. Bob looks at the glimpse of the output Alice viewed, which is exactly what he sees on his execution of the application, and agrees the figure is suspicious. Since he has some background in the structure of this mashup, he decides to inspect the provenance of this output (Fig. 2). In doing this, he compares data retrieved as the result of a SPARQL query to a version of the data formatted for use in the visualization itself. Here, he notices the numerical values in the data record for Afghanistan are multiplied by 1,000, following the process Format Data. Bob concludes that the problem lies with this process, likely due to a unit conversion error, and replies accordingly to Alice that it is an application-specific problem.

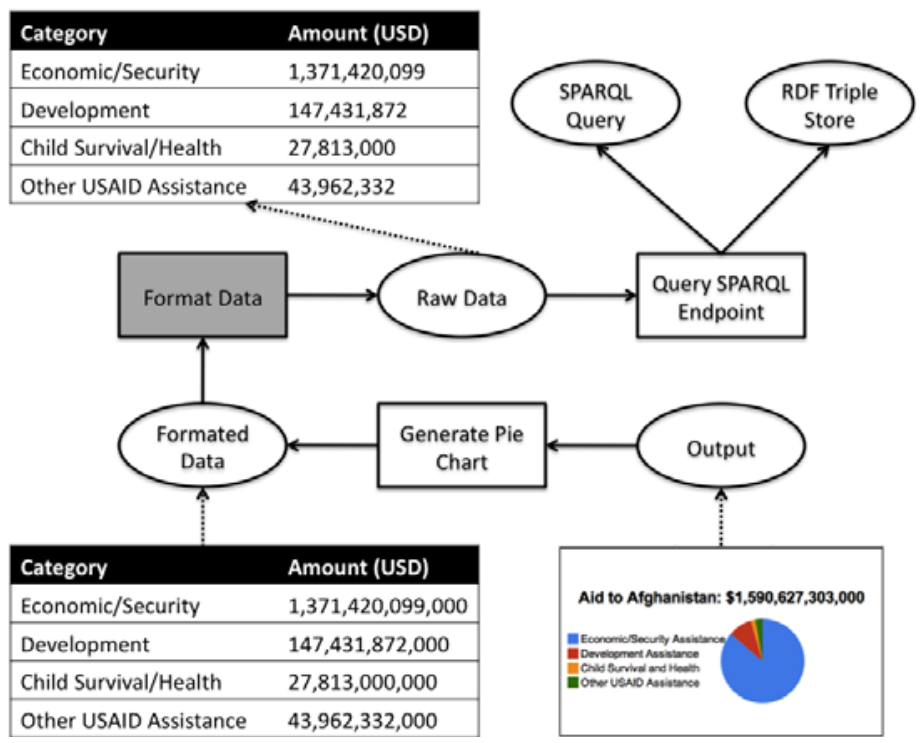

Fig. 2. An OPM-based [9] diagram of the provenance that Bob inspects in Use Case 1, with artifact values illustrated. Here, Bob isolates the problem to the "Format Data" process.

\subsection{Use Case 2: Problem with Data}

Here, another user Carol runs the mashup and sees that Afghanistan had an HDI value of .945 in 2007, which would place it among the world's most developed

\footnotetext{
${ }^{6}$ Approximately 13 trillion, as of June 2010.
} 
countries. Like Alice from Use Case 1, Carol decides against viewing the provenance of this output, instead leaving the comment "How could Afghanistan have an HDI value of .945 in 2007?" Following this, Bob decides to search for comments made on the Afghanistan country facts and finds Carol's comment. Bob looks at the glimpse of the output Carol viewed, but it is different from what he sees - on his screen, Afghanistan's HDI is shown as .345, making it one of the least developed countries. He inspects the provenance of this output (Fig. 3), comparing data retrieved as the result of a SPARQL query to a version of the data formatted for use in the visualization itself. However, the HDI values in both data records are the same - meaning that the HDI value of .945 was obtained as a result of the SPARQL query to DBPedia7. Bob concludes that the problem most likely was with the data on DBPedia, possibly due to a typo or vandalism that was subsequently corrected, and replies to Carol that it is a data-specific problem. 9

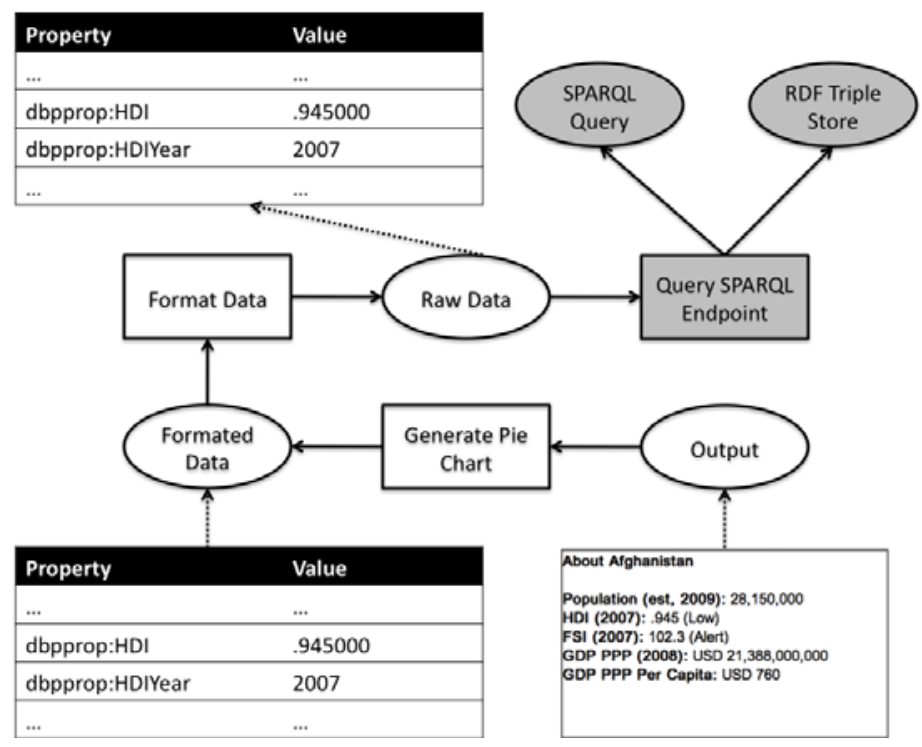

Fig. 3. An OPM-based diagram of the provenance that Bob inspects in Use Case 1, with artifact values illustrated. Here, Bob determines the problem lies before the "Raw Data" artifact.

\section{Discussion and Conclusion}

Currently, the PACT model is defined to express functionality similar to Amazon Mechanical Turk, and therefore faces similar requirements to effectively coordinate the crowdsourcing of tasks. Some of these requirements are discussed in $[$, and include:

\footnotetext{
${ }^{7}$ Here, the mention of any specific error in the DBPedia corpus is hypothetical and done only for illustrative purposes.
} 
- A need for constrained, verifiable problems for crowd members to solve. Else, answers provided by crowd members could simply be a matter of opinion, or guesses as to the answer.

- Determining the incentives for members of a web community to answer questions provided by other users.

- Mechanisms for preventing ineffective or malicious users from registering comments (or replies to comments).

While this paper doesn't discuss the implementation of specific technologies for a PACT, some recommendations are made

- Information Structuring: The goal of this would be to categorize users, as well as the comments/replies they make. Semantic Web languages, such as RDF and OWL, provide such functionality, and can be used in the creation of ontology-based classifications of information. In doing this, functionality

- Viewing Provenance: At present, a number of tools exist for reviewing provenance. Among these are Probe-It and IWBrowser [11, which are designed to view provenance encoded using the OWL-based Proof Markup Language (PML).

Achieving these goals will be important future work for refining the PACT model, as well as for implementing PACT instances in web applications.

\section{References}

1. Berners-Lee, T.: Cleaning up the User Interface (1997), http://www.w3.org/DesignIssues/UI.html

2. Biton, O., Cohen-Boulakia, S., Davidson, S., Hara, C.: Querying and managing provenance through user views in scientific workflows. In: Proceedings of ICDE (2008)

3. Brabham, D.: Crowdsourcing as a model for problem solving: An introduction and cases. Convergence 14(1), 75 (2008)

4. Freire, J., Silva, C., Callahan, S., Santos, E., Scheidegger, C., Vo, H.: Managing rapidly-evolving scientific workflows. Provenance and Annotation of Data, 10-18 (2006)

5. Groth, P., Miles, S., Moreau, L.: A model of process documentation to determine provenance in mash-ups. ACM Transactions on Internet Technology (TOIT) 9(1), $3(2009)$

6. Kittur, A., Chi, E., Suh, B.: Crowdsourcing user studies with Mechanical Turk. In: Proceeding of the Twenty-Sixth Annual SIGCHI Conference on Human Factors in Computing Systems, pp. 453-456. ACM, New York (2008)

7. Miles, S., Groth, P., Deelman, E., Vahi, K., Mehta, G., Moreau, L.: Provenance: The bridge between experiments and data. Computing in Science and Engineering 10(3), 38-46 (2008)

8. Moreau, L.: The Foundations for Provenance on the Web. Foundations and Trends in Web Science (2009)

9. Moreau, L., Clifford, B., Freire, J., Futrelle, J., Gil, Y., Groth, P., Kwasnikowska, N., Miles, S., Missier, P., Myers, J., Plale, B., Simmhan, Y., Stephan, E., den Bussche, J.V.: The open provenance model - core specification (v1.1). Future Generation Computer Systems (July 2010) 
10. Santos, E., Lins, L., Ahrens, J., Freire, J., Silva, C., et al.: VisMashup: Streamlining the Creation of Custom Visualization Applications. IEEE Transactions on Visualization and Computer Graphics 15(6), 1539-1546 (2009)

11. Pinheiro da Silva, P., McGuinness, D., Del Rio, N., Ding, L.: Inference web in action: Lightweight use of the proof markup language. In: Sheth, A.P., Staab, S., Dean, M., Paolucci, M., Maynard, D., Finin, T., Thirunarayan, K. (eds.) ISWC 2008. LNCS, vol. 5318, pp. 847-860. Springer, Heidelberg (2008)

12. Simmhan, Y., Plale, B., Gannon, D.: Query capabilities of the Karma provenance framework. Concurrency and Computation: Practice and Experience 20(5), 441451 (2008)

13. Snow, R., O'Connor, B., Jurafsky, D., Ng, A.: Cheap and fast-but is it good?: evaluating non-expert annotations for natural language tasks. In: Proceedings of the Conference on Empirical Methods in Natural Language Processing, pp. 254263. Association for Computational Linguistics (2008) 